\title{
Plasmonic gold nanostructures for biosensing and bioimaging
}

\author{
Xiaowen Ou ${ }^{1,2} \cdot$ Yuqi Liu ${ }^{1} \cdot$ Mingxing Zhang ${ }^{1} \cdot$ Li Hua $^{1} \cdot$ Shenshan Zhan $^{3}$ (D) \\ Received: 25 February 2021 / Accepted: 14 August 2021 / Published online: 25 August 2021 \\ (C) The Author(s), under exclusive licence to Springer-Verlag GmbH Austria, part of Springer Nature 2021
}

\begin{abstract}
As one kind of noble metal nanostructures, the plasmonic gold nanostructures possess unique optical properties as well as good biocompatibility, satisfactory stability, and multiplex functionality. These distinctive advantages make the plasmonic gold nanostructures an ideal medium in developing methods for biosensing and bioimaging. In this review, the optical properties of the plasmonic gold nanostructures were firstly introduced, and then biosensing in vitro based on localized surface plasmon resonance, Rayleigh scattering, surface-enhanced fluorescence, and Raman scattering were summarized. Subsequently, application of the plasmonic gold nanostructures for in vivo bioimaging based on scattering, photothermal, and photoacoustic techniques has been also briefly covered. At last, conclusions of the selected examples are presented and an outlook of this research topic is given.
\end{abstract}

Keywords Plasmon $\cdot$ Gold nanostructure $\cdot$ Biosensing $\cdot$ Bioimaging $\cdot$ Scattering $\cdot$ Photothermal $\cdot$ Photoacoustic

\section{Introduction}

With the rapid development of nanotechnology, plasmonic metal nanostructures have attracted much attention because of their nanometer size, fascinating physicochemical properties, and surface plasmon behavior [1]. Through modulating their composition, size, shape, and structure [2], the properties and behaviors of plasmonic metal nanostructures could be easily tuned, which guarantee their wide application in fields as diverse as catalysis, energy, environment, electronics, biology, and medicine [3, 4]. Among the various plasmonic nanomaterials, gold nanostructures (including nanospheres, nanorods, nanostars, nanoshells, etc.) have attracted extensive research interest especially in the biology and medicine areas due to their distinct optical and physical properties such as localized surface plasmon resonance (LSPR), Rayleigh

Shenshan Zhan

zhanshenshan@caas.cn

1 Hubei Key Laboratory of Purification and Application of Plant Anti-Cancer Active Ingredients, Department of Chemistry and Life Science, Hubei University of Education, Wuhan 430205, China

2 Research Center for Development and Utilization of Medicinal Plants in Eastern Hubei Province, Hubei University of Education, Wuhan 430205, China

3 Institute of Environment and Sustainable Development in Agriculture, Chinese Academy of Agricultural Sciences, Beijing 100081, China scattering, surface-enhanced fluorescent signal, and surfaceenhanced Raman scattering (SERS) [5-8]. These properties enable gold nanostructures to be a kind of prominent scaffolds for the design of biosensors for a wide range of targets [9-11]. Additionally, gold nanostructures also have many other advantages such as fine biocompatibility, good stability, and diverse functionality, which are ideal for bioimaging and medical applications.

Up to now, various applications in biological sensing, imaging, medical diagnosis, and cancer therapy are based on scattering or absorption of gold nanostructures [12-14]. For instance, the LSPR shift caused by both scattering and absorption and tuned by size, shape, and interparticle distance can be utilized to report the absence or presence of the target molecules [15-17]. Moreover, the strong scattering signal and photostability make them an ideal type of optical labels and probes. In addition, photothermal effect caused by nanostructure absorption is widely used in bioimaging and cancer therapy [18-20]. Its performance depends on a modulated pump laser beam that is employed to heat the plasmonic nanostructure and a probe beam that is used to detect the thermal induced change in the reflective index. Similarly to photothermal imaging, photoacoustic imaging is another technique to detect light absorption but monitoring the resultant acoustic waves. Unlike traditional optical imaging techniques, photoacoustic imaging is noninvasive, and it can monitor acoustic signals upon light excitation, thus detecting tissues deep to about $50 \mathrm{~mm}$ with high temporal resolution and superior selectivity [21]. 
In this review (Fig. 1), a brief overview of the optical properties of plasmonic gold nanostructures for sensing in vitro is firstly introduced, and then the examples of sensing based on localized surface plasmon resonance (LSPR), Rayleigh scattering, surface-enhanced fluorescence, and Raman scattering (SERS) were summarized. Moreover, the progress in which the plasmonic gold nanostructures were used as imaging labels in vivo based on scattering, photothermal, and photoacoustic techniques is also briefly covered. Finally, conclusions of these examples and outlooks on this research topic are proposed.

\section{Plasmonic gold nanostructures for biosensing in vitro}

\section{Sensing based on both absorption and scattering (LSPR shift)}

LSPR is a spectroscopic technique based on the resonant oscillation of conduction electrons at interfaces of noble metal nanoparticles stimulated by incident light. When LSPR happens, some of the photons will be released with the same frequency in all directions, the process of which is defined as scattering. Meanwhile, some of the photons will be transformed into vibrations of phonons or lattice, the process of which is known as absorption [22]. The absorption frequency of the surface plasmon band of metal nanoparticles depends on nanoparticles size, shape, and interparticle distance. Since the LSPR shift based on both scattering and absorption of gold nanostructures (AuNPs) tuned by distance change and structure change of AuNPs can be utilized to report the absence or presence of target molecules, by a visual change of solution color or red shift of the AuNPs' surface plasmon band,

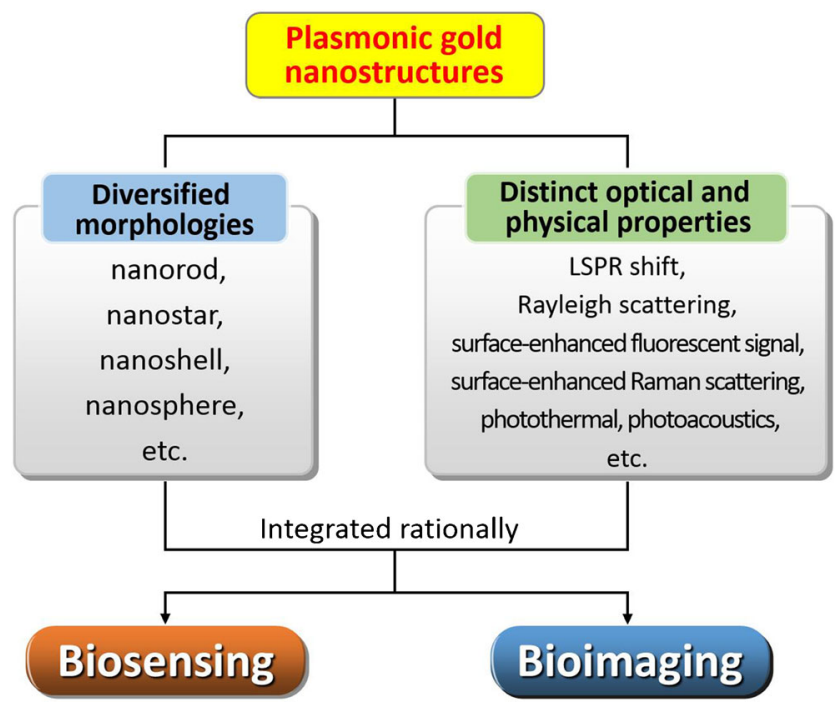

Fig. 1 Overview of this review applications based on LSPR shift were mainly introduced in this section.

\section{LSPR shift based on the distance change}

Plasmonic AuNPs have great promise for use in biomolecular analysis because its optical properties can be used to report the presence and absence of the analytes [23]. Specifically, the change in interparticle separation distances will perturb the LSPR band of the ensemble and lead to a pronounced color variance. Since it was firstly demonstrated for DNA detection by Mirkin et al. in 1996 [24], distance-based LSPR shift has been widely studied for sensing various analytes. For example, Lee and Kang's group reported a colorimetric assay to analyze the biomarker of Middle East respiratory syndrome coronavirus (MERS-CoV) [25]. As shown in Fig. 2A, a pair of DNA sequence modified with thiol at either the $5^{\prime}$ terminal or $3^{\prime}$ terminal to form complementary base pairs with upstream of the E protein gene and open reading frames 1a on MERS$\mathrm{CoV}$ was designed. The target sequence and the two DNA fragment formed a disulfide-induced long self-assembled complex, which protected AuNPs from aggregation induced by salt solution and transition of optical properties. Through LSPR shift and color changes of AuNPs in the UV-vis wavelength range, the platform was able to discriminate $30 \mathrm{bp}$ MERS-CoV down to $1 \mathrm{pmol} / \mu \mathrm{L}$. Taking advantage of the aggregation of AuNPs triggered by liposome encapsulating reagent, a plasmonic enhanced lateral flow assay with an improved colorimetric signal has been proposed [29]. Through this signal enhancement strategy, Escherichia coli $\mathrm{O} 157: \mathrm{H} 7$ in buffer and in liquid food systems could be detected with a limit of detection (LOD) of $100 \mathrm{CFU} / \mathrm{mL}$ and $600 \mathrm{CFU} / \mathrm{mL}$, respectively. Apart from biosensing, the excitation of LSPR of AuNPs can also be utilized for photodynamic therapy. Recently, Wang et al. found that cationic polymer poly (diallyldimethylammonium chloride) (PDDA) could assemble AuNPs form into aggregates with intensive plasmonic resonance [30]. The group also discovered that the triplet state enhancement effect of PDDAassembled AuNPs was sensitive to the size of AuNPs and the concentration of polymer, which exhibit great potential on the applications of AuNPs in triplet exciton harvesting, triplettriplet energy transfer, and photodynamic therapy. Contrast to previous results where only $2-3$ times of the triplet state enhancement effect was displayed [31, 32], the maximum plasmon enhancement effect was calculated to be $\sim 10$-fold detected by transient UV-vis absorption spectroscopy in this report. This obvious enhancement resulted from the plasmon effect of AuNP aggregates shows obvious advantage in establishing sensing method of higher sensitivity.

Besides biomolecular analysis, the AuNP-assisted colorimetric method has also been extended to monitor reaction processes. DNA-functionalized AuNPs are often obtained through thiols labeling; however, the DNA hybridization 


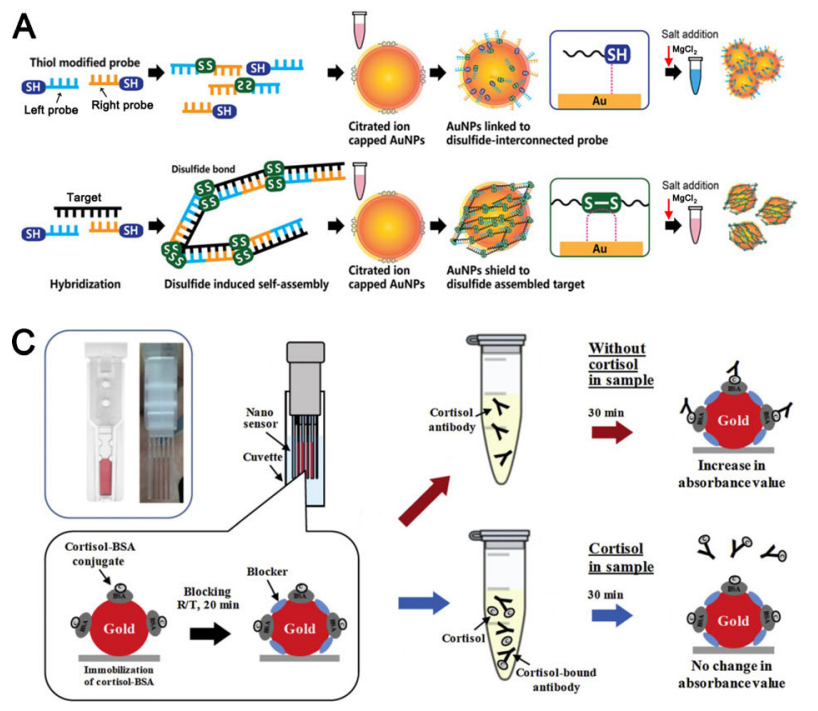

Fig. 2 A The scheme of colorimetric analysis of DNA sequence based on disulfide-induced self-assembly. Reprinted with permission from ref. [25], Copyright 2019 American Chemical Society. B The schematic of specific AuNPs in several kinds of salts solution and the corresponding absorbance. Reprinted with permission from ref. [26], Copyright 2018
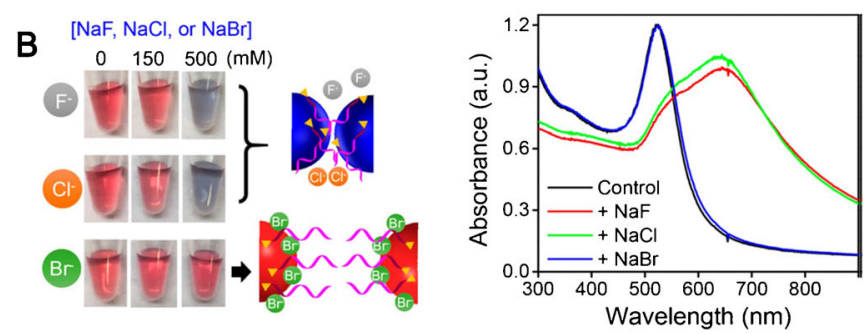

D

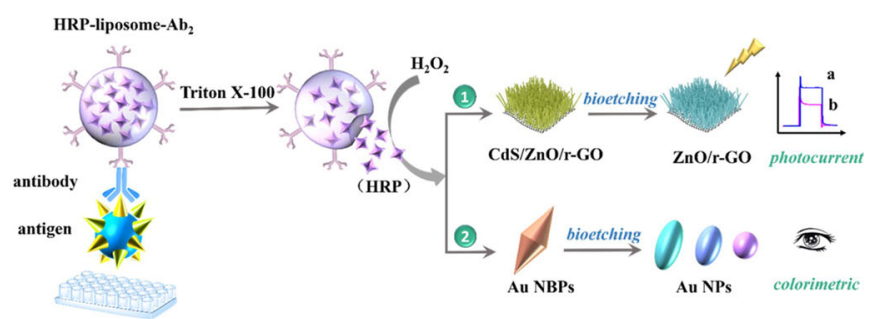

American Chemical Society. C The scheme of the competitive cortisol analysis using PlexNanoCuves. Reprinted with permission from ref. [27], Copyright 2018 Elsevier B.V. All rights reserved. D Schematic illustration of the dual-modality immunosensor construction. Reprinted with permission from ref. [28], Copyright 2019 American Chemical Society would be hindered, and the colloidal stability of the conjugate would be lowered by the inevitable random and uncontrollable DNA base adsorption. Liu et al. discovered that $\mathrm{Br}^{-}$could serve as an effective backfilling agent displacing selected DNA bases on gold [26]. As shown in Fig. 2B, all colorimetric and UV-vis spectroscopy confirmed that $300 \mathrm{mM} \mathrm{NaF}$ and $\mathrm{NaCl}$ could induce dispersed AuNPs to aggregate, while the AuNPs remained dispersed even with $500 \mathrm{mM} \mathrm{NaBr}$. Using these $\mathrm{Br}^{-}$decorated biointerfaces with improved colloidal stability and hybridization efficiency, biosensing and drug delivery benefit from the direct assembly of nanomaterials could be realized. Recently, Rao et al. presented an ingenious strategy for the determination of heavy metal ions, which converted the commonly observed nonspecific turn-off response of [-]AuNP into a dramatic selective turn-on response [33]. Results showed that $\mathrm{Pb}^{2+}$ could break the electrostatic interactions in $[+]-[-]$ AuNP nanoionic precipitates prepared from $11.4 \pm$ $1.2 \mathrm{~nm}$ AuNPs selectively and expel [+]AuNP into solution, leading to the turn on of the plasmonic wine red color. The selectivity of discriminating $\mathrm{Pb}^{2+}$ from other divalent metal ions was attributed to the dominance of interaction energy for the formation of [-]AuNP- $\mathrm{Pb}^{2+}$ complex over the inter-nanoparticle interactions. Compared with the reports based on analyte-specific AuNPs, the relative lower sensitivity of this work may hinder its application. Further efforts on improving the sensitivity of the identification protocol were undertaken by the same group through tuning the interactions in nanoionic precipitates.

\section{LSPR shift based on the structure change}

Apart from the distance change for colorimetric sensing, structure change of AuNPs has also been demonstrated to produce dramatic shifts in LSPR and enhance the detection sensitivity greatly. The binding of a biological molecule onto the surface of gold nanostructure would change the local refractive index and in turn induce a LSPR shift in wavelength. According to this theory, Jeon et al. presented a sensitive and rapid nanosensor for the detection of cortisol hormone [27]. As shown in Fig. 2C, the cuvette-type nanosensor comprised an assembly of plastic unit sensors coating with AuNPs in a single layer, on which cortisol-BSA conjugate was immobilized. The above conjugate could bind cortisol antibody and induced a red shift in LSPR wavelength. As a proof of concept, cortisol both in PBS solution and serum over the range of $2.759-3 \times 10^{3} \mathrm{nM}$ could be detected within only $20 \mathrm{~min}$. Funari et al. [34] developed a label-free sensing platform consisting of a gold nanospike covered glass substrate to detect the presence of antibodies against the SARS-CoV-2 spike protein, based on the LSPR peak shift of gold nanospikes. This platform was fabricated by gold electrodeposition and integrated in a microfluidic chip coupled with a reflection probe. A series of comparative experiments displayed that $\mathrm{Au}$ nanospikes with a surface coverage of $\sim 20 \%$ have the strongest light absorption at $\sim 513 \mathrm{~nm}$. The LSPR shift degree was correlated with the concentration of target antibody bound with antigen, and a LOD of $\sim 0.5 \mathrm{pM}$ could be achieved within $30 \mathrm{~min}$. Through applying the 
phenomenon that benzoyl peroxide (BPO) could oxidize Ag nanoshells on the surface of Au@Ag nanorods (NRs), Lin et al. presented a sensitive colorimetric assay of high resolution for the detection of BPO [35]. In contrast to AuNPs, the isotropic AuNRs possess longitudinal LSPR peaks that are highly sensitive to the change of their aspect ratio. The etching of Ag nanoshells could change the aspect ratio of AuNRs and cause a red shift of longitudinal LSPR peak, leading to a sharply contrasting multicolor change. The stick-like AuNRs with $15 \pm 3 \mathrm{~nm}$ in diameter and $50 \pm 8 \mathrm{~nm}$ in length exhibit two extinction bands around $515 \mathrm{~nm}$ and $820 \mathrm{~nm}$, respectively. The red shift of longitudinal LSPR peak was linear with the $\mathrm{BPO}$ at concentrations ranging from 0 to $100 \mu \mathrm{M}$ with a LOD of $0.75 \mu \mathrm{M}$. Additionally, Au nanobipyramids (AuNBPs) with sharp edges are expected to be ideal colorful chromogenic substrates due to their high sensitivity to refractive index. Based on the LSPR peak shift of AuNBPs that have a standard bipyramid shape with a brown color and the photocurrent response of flexible reduced graphene oxide or $\mathrm{CdS} / \mathrm{ZnO}$ NR arrays, Wei et al. [28] developed a dual-modality readout immunoassay platform for the broad-spectrum and sensitive determination of ochratoxin (Fig. 2D). In this assay, nanoliposomes were used to encapsulate horseradish peroxidase (HRP) and as the carrier of the secondary antibody. In the presence of $\mathrm{H}_{2} \mathrm{O}_{2}$, the HRP could trigger enzymatic bioetching of $\mathrm{CdS}$ on the photoelectrode, producing a detectable change of photocurrent. Moreover, HRP could oxidize $\mathrm{H}_{2} \mathrm{O}_{2}$ to generate hydroxyl radicals to etch AuNBPs, which would induce LSPR shifts and multiple color changes (brown $\rightarrow$ gray green $\rightarrow$ bluish green $\rightarrow$ pink). Compared with the previous reports, this immunoassay exhibited a much lower LOD due to the greatly photocurrent and peak shift signal amplified [36, 37]. What is more, owing to the completely independent transmission mechanism and signal conversion, this dual-modal immunoassay was more reliable and precise as compared to the corresponding single-modal immunoassay.

\section{Sensing directly based on Rayleigh scattering}

Sensing based on both absorption and scattering (LSPR shift) is attractive for diagnostic applications. However, measuring a whole LSPR spectrum of individual particle typically requires long integration time for a regular spectrometer. Moreover, optical modeling and experimental data demonstrated that a great many of pony-size gold particles (e.g., about $15 \mathrm{~nm}$ in diameter) are needed to provide a measurable red shift in the surface plasmon band. Hence, directly monitoring scattered light rather than reflected light from AuNPs of large size (e.g., about 40-100 $\mathrm{nm}$ in diameter) would improve the sensitivity of sensors. When the large size AuNP was brought to the immediate distance of another one, the two plasmonic nanoparticles would occur coupling effect, resulting in dramatic intensity amplification and distinctive shift of the scattering spectra. Compared with the aggregation induced red shift in LSPR mentioned above, Müller et al. found that the color of scattered light changed concomitantly and provided a more sensitive means of detecting nanoparticle hybridization complexes when spotted onto a waveguide [38]. By taking advantage of this coupling effect, Zhai et al. [39] presented a selective and sensitive detection and imaging of $\mathrm{Cu}^{2+}$ in live cells at a single-particle level. As shown in Fig. 3A, a core-satellite plasmonic nanoprobe, of which the shift in plasmon resonance wavelength and the scattering cross section will be significantly altered by the satellite number, the satellite radius, the core radius, and the distance between core and satellites, was assembled by metal ion-dependent DNA-cleaving DNAzyme linker. In the presence of $\mathrm{Cu}^{2+}$, the DNAzyme linker would be cleaved, induce the disassembly of the nanoprobes, and result in visible color change of the scattering light and unique blue shift of the scattering spectra of gold core-satellite probes. In this system, the LOD was as low as $67.2 \mathrm{pM}$, and the nanoprobes were further applied for intracellular $\mathrm{Cu}^{2+}$ imaging analysis. Since the disassembly of the $\mathrm{Au}$ core-satellite probes could lead to dramatic blue shifts of the scattering spectra as well as naked color change, this coupled plasmonic nanoassembly offers a much more excellent detection sensitivity compared with the conventional $\mathrm{Cu}^{2+}$ sensing methods based on colorimetric and fluorescent analysis $[43,44]$. Lee et al. also reported the disassembly of core-satellite nanostructure for protease activity detection by taking advantage of the change of light scattering [45]. The negatively charged AuNPs of $50 \mathrm{~nm}$ which served as a core of the nanosensor were fixed on the positively charged surface of the 3-aminopropyltriethoxysilane-functionalized glass slide. Then the biotinylated peptides were attached onto the surface of core nanoparticles via Au-thiol contact. After that, the coresatellite nanosensor was self-assembled by the binding between the streptavidin-modified satellite and the biotinylated peptide. As a result, the complete color change originated from the trypsin-induced disassembly of core-satellite nanostructure could be captured by a true color charge-coupled device camera. Apart from core-satellite structure, Zhao et al. presented a new strategy for miRNA analysis based on the formation of a plasmonic Au@Ag nanosnowman (Fig. 3B) [40]. The plasmonic Au@Ag nanosnowman was obtained through integrating hybridization chain reaction (HCR) amplification and exonuclease III (Exo III)assisted target recycling strategy. The Au@Ag head-body structure with greatly changed scattering wavelengths was formed because of the asymmetrically deposition of the $\mathrm{Ag}^{+}$released by dissolved AgNCs on $50 \mathrm{~nm}$ AuNPs. In this way, miRNA-21 could be detected with a proportional band from $1 \mathrm{fM}$ to $100 \mathrm{pM}$, as well as a LOD of $0.60 \mathrm{fM}$ which is much lower than previous reports [46]. Through applying dark-field microwells and $50 \mathrm{~nm}$ AuNPs that functionalized 
A

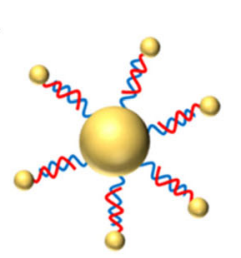

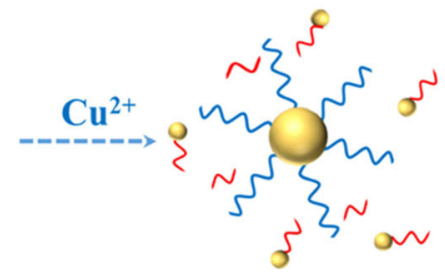

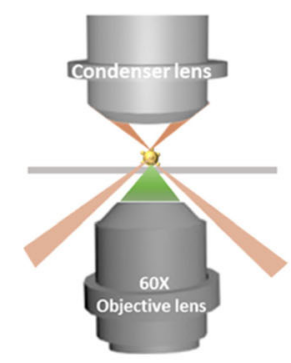

C

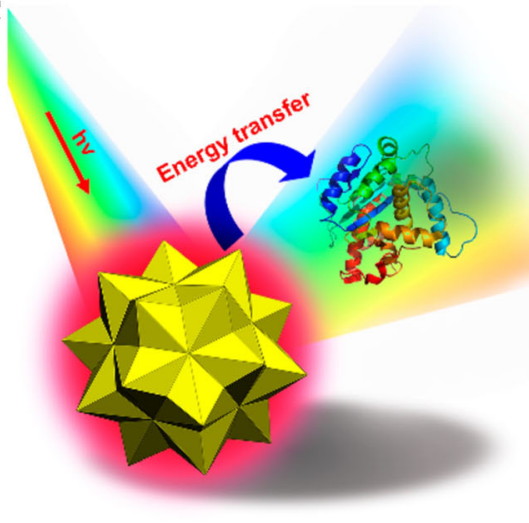

B
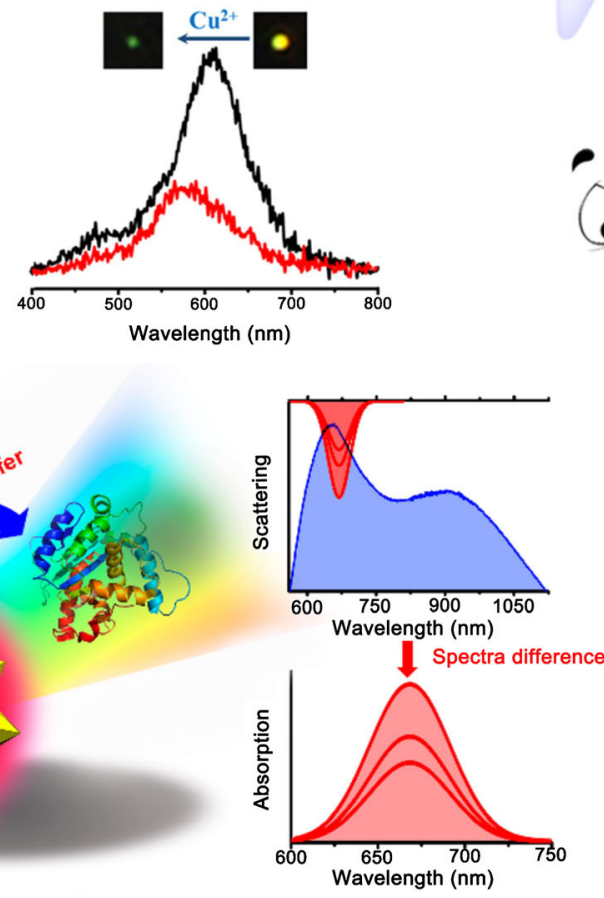
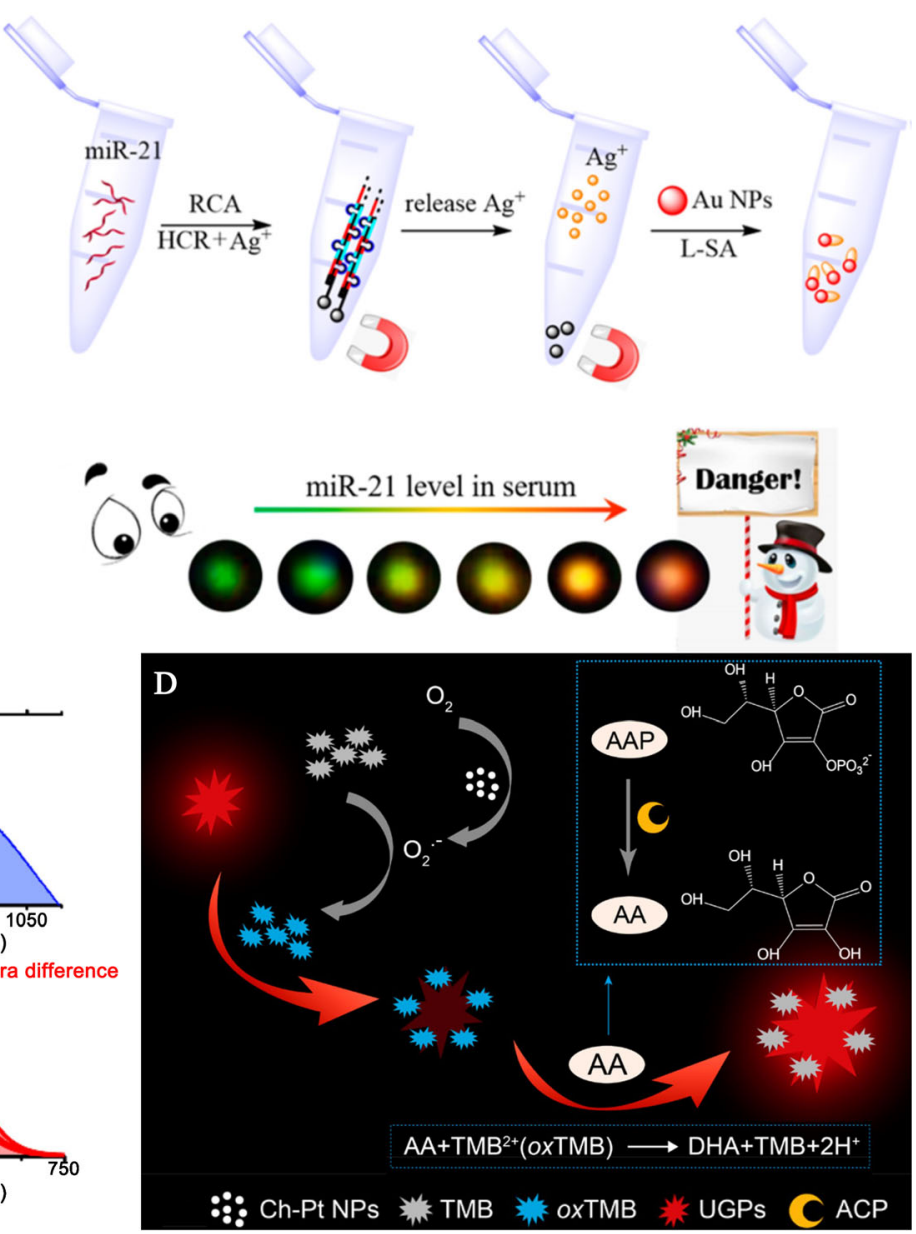

Fig. 3 A Schematic of the scattering spectrum of Au core-satellite nanoassembly before and after incubation with $\mathrm{Cu} 2+$. Reprinted with permission from ref. [39], Copyright 2018 American Chemical Society. B Schematic illustration of the principle for determination of miRNA-21 (miR-21). Reprinted with permission from ref. [40], Copyright 2019 American Chemical Society. C The scheme of the specific biomolecule analysis and enzyme kinetics based on plasmon resonance energy transfer (PRET) spectroscopy on single gold nanonstars. Reprinted with permission from ref. [41], Copyright 2018 American Chemical Society. D Schematic illustration of the acid phosphatase detection based on PRET. Reprinted with permission from ref. [42], Copyright 2020 American Chemical Society

quenching in Rayleigh scattering, which only occurs if the AuNPs and the target have a matched frequency. Since firstly been discovered in 2007, the PRET has been widely utilized for molecules recognition and ions detection [49]. For instance, Chen's group applied PRET-based spectroscopy on single gold nanostar (AuNS) to study the enzyme and specific biomolecule recognition kinetics [41]. In this assay, anisotropic AuNSs were applied due to the possibility of fine-tuning their optical properties through altering the degree and size of branching. The average size of the involved symmetric AuNSs is $\sim 408 \mathrm{~nm}$, the average diameter of their icosahedral gold seeds is $\sim 200 \mathrm{~nm}$, and the plasmon resonance peak is around $575 \mathrm{~nm}$. As shown in Fig. 3C, the kinetics of DNase I digestion and biotin-streptavidin recognition in different physiological environment were explored via detecting the variances of absorption spectra for the black hole quencher ${ }^{\circledR} 3$ (BHQ-3), and results show that the sizes of molecules and the viscosities of media play a critical role in enzyme digestion 
and biomolecule recognition. Liu et al. discovered PRET from a gold nanosphere (with a diameter of $70 \mathrm{~nm}$ and a scattering peak ranging from 490 to $690 \mathrm{~nm}$ ) to a quantum dot at the single-particle level [8], and through which, a separation-free homogeneous immunoassay for prostate-specific antigen with an ultralow LOD of $0.2 \mathrm{fM}$ was developed. Urchin-like AuNPs (UGP) that have unique optical properties compared to the corresponding spherical AuNPs of the same core diameter have also been applied. It can cause a red shift in the surface plasmon peak and a larger enhancement of the electromagnetic field $[42,50]$. Taking advantage of the PRET between oxTMB and UGPs with a central core and multiple sharp tips and a mean size of $138.4 \mathrm{~nm}$, Yan et al. designed a new approach for acid phosphatase (ACP) detection (Fig. 3D) [51]. When the ACP was introduced, 2-phospho-L-ascorbic acid trisodium salt (AAP) could be converted into hydrolyzate ascorbic acid (AA), which would reduce oxTMB into TMB, thus inhibiting the emergence of PRET and recovering the scattering spectral intensity of UGPs. Through this strategy, ACP could be monitored with a LOD of $0.076 \mathrm{U} / \mathrm{L}$. Wang et al. designed a kind of "loading-type" plasmonic nanomaterials [52], which constituted of mesoporous silicon-coated AuNPs (Au@MSN, diameter $\sim 60 \mathrm{~nm}$ ) as PRET donors and loaded organic small molecules (termed as $\mathrm{CHCN}$ ) as PRET acceptors. Since the $\mathrm{CHCN}$ has an absorption band at $560 \mathrm{~nm}$ and indicates a strong overlap with the LSPR of Au@MSN, the PRET process between them was guaranteed. In the presence of the target $\mathrm{ONOO}^{-}$, the $\mathrm{CHCN}$ specifically reacted with $\mathrm{ONOO}^{-}$, generated a product, and blocked the PRET. In this way, the "loading-type" plasmonic nanosensor could realize the $\mathrm{ONOO}^{-}$determination with good selectivity and sensitivity (at a single nanoparticle level). Since PRET has broad applications in biosensing, the effect of the distance (d) between the conjugated molecules and the plasmonic nanoparticle on the PRET efficiency ( $\eta$ PRET) has been investigated by utilizing Cy 3 molecules or tetramethylrhodamine as acceptors and single spherical AuNPs as donors [53]. Double-stranded DNA was applied to regulate the $d$-values between the donor and the acceptor. Both theoretical and experimental results show that a decay function dependent on $d$-value for the $\eta$ PRET could be obtained, which would shed light on more PRET-based biochemical sensors.

\section{Sensing based on surface-enhanced fluorescent signal}

Interaction between the excited state fluorophores and free electrons on the plasmonic metal surface significantly affects fluorescent intensity. For example, in fluorophore quenching at $0-5 \mathrm{~nm}$, the incident light field occurs at a spatial variation of $0-15 \mathrm{~nm}$, and the radiative decay rates change at $0-20 \mathrm{~nm}$ [54]. The localization of fluorophores near metal nanoparticle will lead to dramatic fluorescent signal enhancement on the nanometer scale and allows for an even lower LOD for early disease detection and clinical diagnosis applications. Gao et al. constructed a versatile AuNSs@ $\mathrm{SiO}_{2}$-based plasmonenhanced fluorescence probe together with 20 symmetric "hot spots" for in situ "lighting up" imaging of intracellular miRNAs [55]. By regulating the thickness of the silica shell, the distance between AuNSs and fluorescent dyes was controlled, and an optimum fluorescence enhancement (21-fold) was obtained when the silica shell was of approximately $22 \mathrm{~nm}$ thickness. As shown in Fig. 4A, the assistant probe, which was in fluorescent "off" state owing to the FRET between Cy5.5 and BHQ-3, was bonded to the AuNSs@ $\mathrm{SiO}_{2}$ to form the enhanced fluorescence probe. The miRNA-21 could hybridize with the Cy5.5 labeled sequence (assistant DNA2) completely and replace the BHQ-3 labeled sequence (assistant DNA1), inducing Cy5.5 far away from BHQ-3 and "turn on" the fluorescent signal. Because of the involvement of these 20 powerful "hot spots" which could produce stronger localized electric fields, this AuNS probe exhibited stronger plasmonenhanced effects than the traditional plasmonic nanostructures, and a LOD of $0.21 \mathrm{pM}$ which is much lower than those of recently reported approaches was achieved [59,60]. What is more, the probe could also discriminate cancer cells and normal cells by different expression levels of miRNA- 21 . Since there are rare research reports on experimental evidence for enhanced fluorescence from dyes bound to AuNSs, Zhou et al. presented a groundwork of two-photon induced fluorescence enhancement of $\mathrm{Cy} 3$ near AuNSs in colloids [61]. By controlling the ratio of $\mathrm{Cy} 3$ signal on the AuNSs via changing the nanoassembly synthesis conditions and a biotin displacement experiment, enhancement factors ranging from 1.2 to 3.5 were obtained. Using immunosensor based on metalenhanced fluorescence, Miranda et al. [62] realized the immunoglobulins analysis in a common antigen-antibody model. In this assay, the gold nanostructures arrays which were constructed by a simple and efficient three-step process were adjusted by modulating the size, interparticle distance, and optical properties of AuNPs. Optimal fluorescence enhancement ( $\sim 150$ times) of this immunosensor was achieved, and the LOD was determined as $4.3 \mathrm{ng} / \mathrm{mL}$. Since this assay exhibit comparable LOD to the standard techniques to quantify proteins in biological fluids like nephelometry [63], it possess great promise for real sample analysis in the future.

For the past 2 years, extraordinary efforts have been made to reveal whether the surface-enhanced fluorescent signal could be exploited advantageously to overcome the drawback of photobleaching and weak fluorescence associated with traditional organic dyes. Peng et al. presented a nanogap antenna-based sensing platform to detect the low-abundance nucleic acid biomarkers with high sensitivity [56]. AuNRs with an aspect ratio of 2.25 (length $=27 \pm 4 \mathrm{~nm}$, width $=$ $12 \pm 2 \mathrm{~nm}$ ) that generated a longitudinal plasmonic 


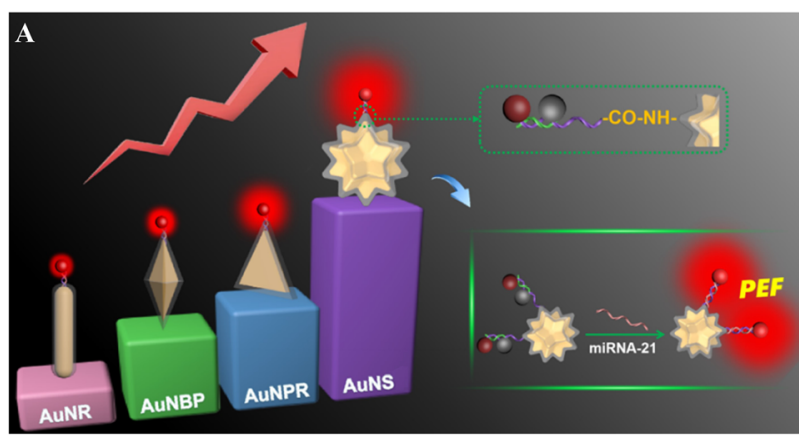

C

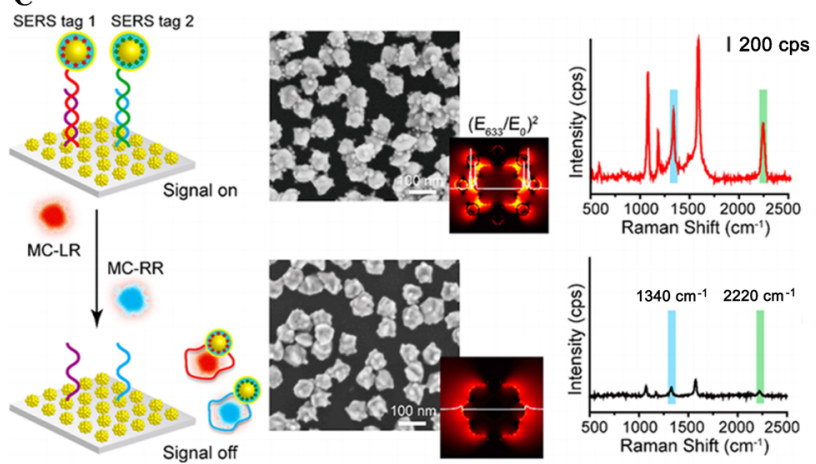

Fig. 4 A The scheme of the AuNSs@ $@$ SiO2-based probe for "lighting up" detection of miRNA-21. Reprinted with permission from ref. [55], Copyright 2021 American Chemical Society. B Schematic representation of the nanogap antennas assembly for target miRNA detection. Reprinted with permission from ref. [56], Copyright 2020 WILEY-VCH Verlag GmbH \& Co. KGaA, Weinheim. C The scheme

wavelength at $660 \mathrm{~nm}$ were utilized. As shown in Fig. 4B, two DNA hairpin sequences which could complementary hybridize with the opposite ends of the target molecules were immobilized on the tips of AuNRs through Au-S bonds. The competition result between the FRET and fluorescent signal enhancement depended on the distance between the fluorophore and the AuNR surface. In the existence of targets, end-to-end AuNR dimers were assembled and the fluorophores quenched by the AuNRs exhibited a dramatic fluorescence enhancement. As a result, miRNA-21 could be detected with a low LOD of $97.2 \mathrm{aM}$, which is $\sim 10$ times lower than those of recently reported other methods [64]. Theodorou et al. reported an AuNSs monolayers assembled plasmonic arrays which enabled up to 19-fold fluorescence signal enhancement for cellular imaging in the near-infrared (NIR) biological window, allowing the application of low quantum yield fluorophores for sensitive bioimaging [65]. Compared with those commercial SPR chips that lack systematic LSPR tuning, these AuNS arrays exhibit great potential for multiplexed applications [66]. Luan et al. introduced a stable and bright fluorescent nanoconstructure, termed plasmonic-fluor, which exhibits up to $6700( \pm 900)$-fold brighter signal compared with the corresponding single NIR fluorophore [67]. By this ultrabright fluorescent enhancement,
B

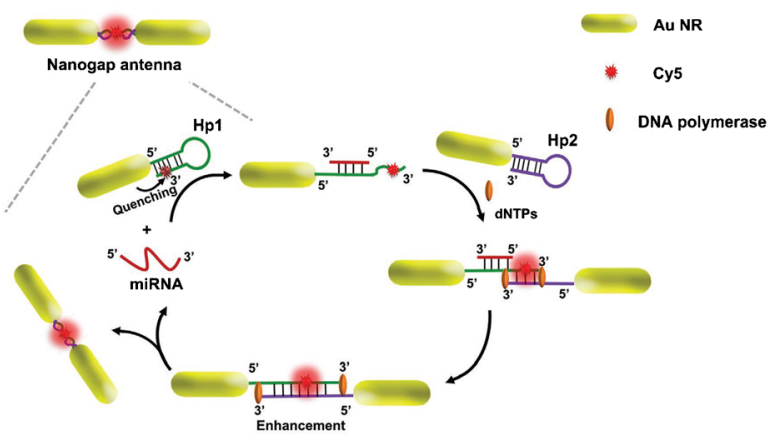

D

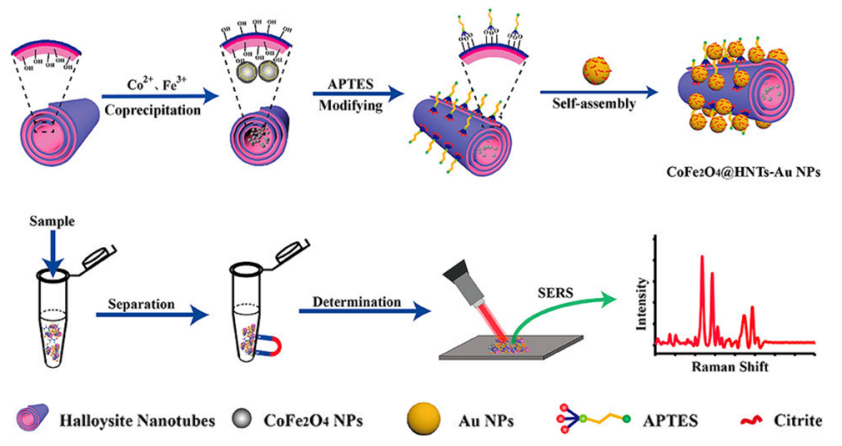

of multiplexed SERS detection of microcystins with aptamer-driven coresatellite assemblies. Reprinted with permission from ref. [57], Copyright 2021 American Chemical Society. D The scheme of the synthesis process of CoFe2O4@HNTs/AuNPs composites. Reprinted with permission from ref. [58], Copyright 2020 American Chemical Society

femtomolar LOD of analytes with standard bioassays could be achieved. Chakkarapani et al. developed a simple and reliable method that provided up to $\sim 100$-fold uniform fluorescence enhancement of endogenous fluorescence of the capsaicinoid molecule [68]. Through monitoring the endogenous fluorescence of capsaicinoid enhanced by plasmon single nanoparticles, ultralow LOD of zM $\left(10^{-21} \mathrm{M}\right)$ level which was $10^{6}$ times lower than that in previous reports was obtained by this method [69]. Since no additional toxic labeling probes were required, this method pioneers a novel strategy for endogenous fluorescence detection.

\section{Sensing based on surface-enhanced Raman scattering}

Surface-enhanced Raman scattering (SERS) phenomenon can be observed when molecules were adsorbed onto or near a plasmonic nanostructure. SERS is a highly sensitive and specific technique because it can detect analyte at molecular level and identify molecules according to their unique vibrational energy levels. More importantly, unlike spectroscopic technique based on Raleigh scattering, which requires a particular absorption wavelength of analyte, SERS has a much wider application due to Raman fingerprints [70, 71]. For instance, Luo et al. developed a SERS aptasensor which could 
individually or simultaneously detect microcystin (MC) [57]. Two of the most commonly encountered species in natural water or in algal culture, MC-LR and MC-RR, were determined as model case. As shown in Fig. 4C, the sensor was constructed by the hybridization of capture-functionalized $\mathrm{Au}$ nanoflowers (AuNFs) dispersed on the substrate with aptamer-functionalized Au@label@Ag@Au SERS tags. These tags of a double shell exhibit two remarkable advantages: (i) the silver layer not only intensifies the plasmonic enhancement but also protects the labels from environmental interference, and (ii) the outer gold coating prevents the oxidation of silver and allows for greater biocompatibility. In the presence of MC-LR and/or MC-RR, the aptamerfunctionalized composite structure dissociate from the AuNFs due to the stronger affinity of the aptamers with the MCs, which decreases the SERS signal. Compared with other recently reported SERS-based strategies for MC-LR analysis [72], the LODs of this SERS aptasensors have been significantly decreased for separate MC-LR detection $(0.8 \mathrm{pM})$ and for multiplex detection (1.5 pM for MC-LR and $1.3 \mathrm{pM}$ for MC-RR) partly attributed to the plasmonic enhancement effect of AgNPs. Although SERS technique is capable of detection analyte at molecular level, there is still a challenge to analyze samples with sensitivity and accuracy due to the large variations in molecular conformation and/or packing density. In order to improve the detection reliability, Feng et al. developed a novel dual-mode AuNPs probe based on SERS and colorimetry for sensitive detection of telomerase activity both in cell extracts and in the urine sample of patients [73]. In their design, a high uniform Au dimer-based probe, in which the single AuNP was $13 \pm 2 \mathrm{~nm}$ and the gaps of Au dimers were $\sim 2 \mathrm{~nm}$, was successfully assembled, and telomerase activity was indicated by the color changes of solution and Raman intensity of the Raman reporter. Through applying the dualmode of SERS and colorimetric techniques, determination of the telomerase activity with good sensitivity $\left(6.1 \times 10^{-15} \mathrm{IU}\right)$, accuracy, and wide range was achieved. Except for the detection of a single substance, Zhang et al. [58] filled magnetic $\mathrm{CoFe}_{2} \mathrm{O}_{4}$ beads inside the halloysite nanotubes (HNTs) and designed a $\mathrm{CoFe}_{2} \mathrm{O}_{4} @ \mathrm{HNTs} / \mathrm{AuNPs}$ substrate for fast and effective determination of 4,4'-thiodianiline and nitrofurantoin in real samples all-in-one by magnetic solid-phase extraction SERS, and the LODs were $0.026 \mathrm{mg} / \mathrm{L}$ and $0.014 \mathrm{mg} / \mathrm{L}$, respectively (Fig. 4D). Choi et al. [74] prepared Au hollow nanospheres with size of $38 \mathrm{~nm}$ and wall thickness of $10 \pm 3 \mathrm{~nm}$ and synthesized PEGylated Ag-encapsulated $\mathrm{Au}(\mathrm{Ag}-\mathrm{Au}$ ) hollow nanospheres to develop a reproducible, stable, and sensitive SERS nanotags for the distribution location of biomarkers. As a result, localized distributions of three cancer biomarkers including EpCAM, ErbB2, and CD44 expressed on breast cancer cells could be accurately identified.

Since the SERS spectrum is obtained in the light-excited area, the enhanced effect may vary with the position of the substrate, and it is crucial to match the enhanced area with the illuminated spot. In order to address this problem, Hong et al. [75] developed a novel technique combining label-free SERS with plasmonic trapping, by which AuNPs could be collected in the center of initially manufactured nanobowtie structures where hot spots are formed when a laser was excited. In this way, rhodamine $6 \mathrm{G}$ low to $100 \mathrm{pM}$ could be detected, and the repeatability could be further improved through calculating the trapping force, electric field distribution, and trapping potential. Kuttner et al. [76] reported the synthesis of gold nanotriangles (AuNTs) in a seedless way using benzyldimethylammonium chloride (BDAC) and 3-butenoic acid (3BA). The preformed 3BA-synthesized AuNT seeds could overgrown up to a final thickness of $80 \mathrm{~nm}$ and an edge length of $175 \mathrm{~nm}$ while maintaining their tip sharpness and triangular shape. Based on the size-dependent SERS performance of AuNTs, 4-mercaptobenzoic acid and BDAC at nanomolar concentrations $\left(10^{-8}-10^{-9} \mathrm{M}\right)$ could be monitored. This sensitivity is in the same order of magnitude as for AuNTs obtained from a previously reported seed-mediated approach [77].

\section{Plasmonic gold nanostructures for bioimaging in vivo}

Compared with most of the organic fluorophores utilized for in vivo cellular imaging, the plasmonic gold nanostructures possess several advantages because of their water solubility, biocompatibility, and resistance to photobleaching and blinking. These advantageous properties make them appropriate for long-time observation in vivo [78]. In the following sub-sections, bioimaging in vivo by scattering, photothermal, and photoacoustics of plasmonic gold nanostructures was summarized.

\section{Bioimaging based on scattering}

The electromagnetic fields will be significantly enhanced especially in the interstitial spaces between two or more interacting particles formed by hybridization of DNAmodified AuNPs. When the interparticle spacing of a dimer is less than $3 \mathrm{~nm}$ by binding to a single target molecule, the signal intensity of the single dimer would be strong enough to permit intracellular imaging. As a pioneer in this field, Lee et al. [79] developed a hyperspectral imaging strategy based on plasmonic dimer probes for the determination and quantification of mRNA splice variant in individual living cells. In their strategy, DNA strands complementary to the specific sequences of BRCA1 mRNA were labeled to $40 \mathrm{~nm}$ AuNPs. After hybridizing with a single target mRNA, an AuNP dimer was formed, which would lead to a spectral peak shift because of strong plasmonic coupling. Using dark-field hyperspectral 
images, single mRNA could be successfully tracked and the distribution of three selected splice variants of the target mRNA could be monitored. Since this study provides insights into RNA and its transport in living cells, it could improve understanding of pharmacogenomics, genetic diagnosis, and gene therapies [80]. Recently, the same group also reported a strategy for live cell imaging by constructing a quantum biological electron tunneling (QBET) junction that permits to observe the behaviors of electron tunneling by distinct barrier widths of barrier molecules [81]. As shown in Fig. 5A, cytochrome c (Cyt c) was bonded to a AuNP of $\sim 50 \mathrm{~nm}$ by a linker molecule to form a quantum tunnel junction with a potential barrier. The AuNPs served as optical antennas that enable Cyt c electron transfer (ET) imaging and the QBET dynamics transmitting in living cell. Based on the AuNP optical antennas, Cyt c ET in redox dynamics process during cellular necrosis and apoptosis in live cells could be captured at the molecular level in real time via QBET spectroscopy. It is hoped that the direct observation of quantum electron tunneling demonstrated in this study would provide new insight into the quantum mechanisms in regulating cellular processes as well as life and death [86]. In addition, through a nanoparticlemediated Raman imaging method, Cho et al. [87] realized the noninvasive analysis and selective isolation of circulating cancer stem cells (CCSCs). In their method, an integrated optimization Raman-active nanoprobe (RAN) platform was built by combining a micro-fluidic chip to manipulate the complete blood samples. By these entirely different Raman signals of RANs, circulating tumor cells (CTCs) and CCSCs were discriminated (90\% efficiency), and classification of these two cells was achieved according to their respective surface marker expression.

\section{Bioimaging based on photothermal}

For single molecular detection in live cells, the plasmonic gold nanostructure should be large enough to be detectable via Rayleigh intensity scattering. However, the size of the nanostructures may sterically hamper the interaction between the labeled molecules. On the other hand, despite the scattering properties are useful for tracking plasmonic nanoparticles, the methods based on absorption may provide stronger signal than those based on scattering [88]. In this context, Lasne et al. [89] proposed a photothermal imaging method for tracking individual AuNP in live cells by combining the advantages of tiny nanometer-sized labels and long observation times. In this research, individual AuNPs of $5 \mathrm{~nm}$ in living cell were tracked owing to the photothermal effect and monitoring of the laser-induced scattering around a NanoAbsorber. Furthermore, recordings of the long trajectories of glutamate receptors on the plasma membrane of live neurons were also demonstrated. Recently, using lightabsorbing AuNPs as nanosources of heat and quadriwave lateral shearing interferometry (QLSI) measurements based temperature mapping technique, Robert et al. [82] demonstrated the laser-induced dynamical control of the heatshock response at single cell level (Fig. 5B). Since the QLSI is a label-free microscopy imaging technique, this approach was noninvasive, and there was no constraint limiting the choice of fluorescent labels for the monitor of the cell's response. Moreover, the QLSI is fast (a few seconds per image or even less), and the use of AuNPs as the absorbing medium offers several benefits such as size tunability and fabrication convenience (can be fabricated using a simple chemical approach). It is anticipated that this study could favor the development of a nascent field of research called thermal biology at the single cell level [90]. Besides these aforementioned techniques, photothermal imaging (PhI) microscopy is another label-free optical microscopy technique that can image the photothermal property of single nanoparticles and nano molecules. Shibu et al. [91] reported the synthesis, sorting, and characterization of monodisperse AuNRs, which show additional red shifted plasmon resonance compared with their spherical counterparts. A dual color PhI microscope was applied for imaging the AuNR at single level and demonstrating that AuNRs are promising basic building blocks for the cellular imaging of biomolecules in the region of NIR.

In addition to gold nanospheres and AuNRs, gold nanoshells also provide appealing characters for bioimaging. Through adjusting the core radius and/or the shell thickness, the optical resonances of nanoshell can be tuned in the region of NIR, where the light can penetrate through tissues maximally because of the low absorption and scattering from intrinsic chromophores [92]. What is more, the large optical cross sections and rigid metallic structures also make the gold nanoshell a promising tool in vivo imaging. For example, Liu et al. [93] fabricated a kind of nanoshell which is composed of $\mathrm{MoS}_{2}$ core that covered by a thin gold shell that formed from gold nanostructures of the average thickness of $4.4 \pm 0.6 \mathrm{~nm}$ and the diameter of $39 \pm 13 \mathrm{~nm}$. With the protection of templated $\mathrm{MoS}_{2}$ nanosheets, the $\mathrm{MoS}_{2}-\mathrm{Au}$ nanostructures show great photostability. Moreover, these as-prepared $\mathrm{MoS}_{2}$-gold nanostructures with strong photoelectric absorbance and enhanced NIR absorbance could be used as a multifunctional theranostic agent with significant potential for dual-modal imaging-guided photothermal-radiation therapy of cancer. Since the core-shell structure and the aspect ratio could tune photothermal conversion efficiency $(\eta)$ of gold nanostructures, Ma et al. [83] developed an easy method for the synthesis of core-shell AuNR@LDH nanostructure which combined layered double hydroxides (LDHs) with AuNRs of the average length of $\sim 60 \mathrm{~nm}$ and the aspect ratio of $4: 1$ (Fig. 5C). Since the interaction between Au and LDHs could result some electron deficiency on the surface of $\mathrm{Au}$, and more electrons could induce more thermal energy conversion, the $\eta$ value of 
A (a)
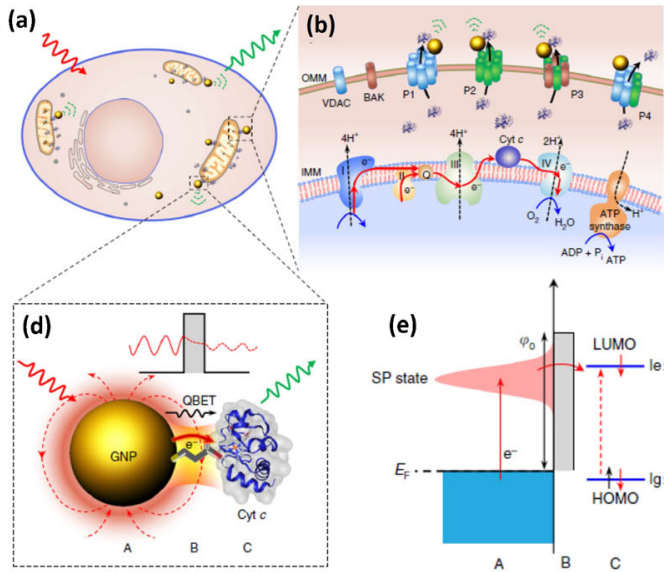
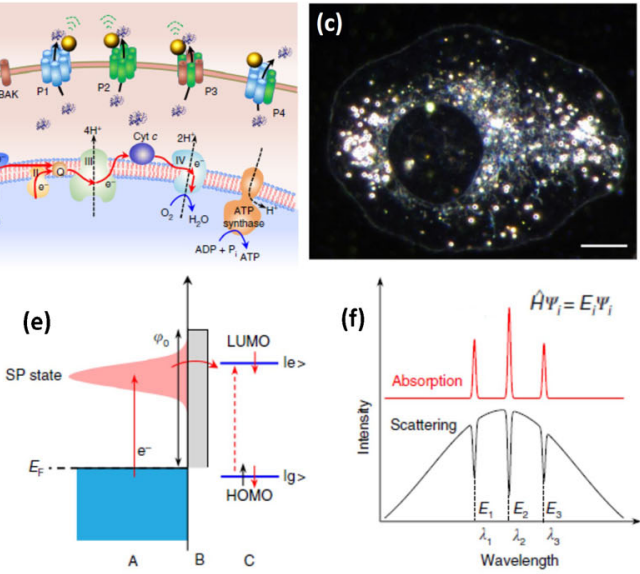

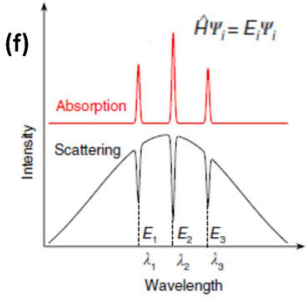

B (a)
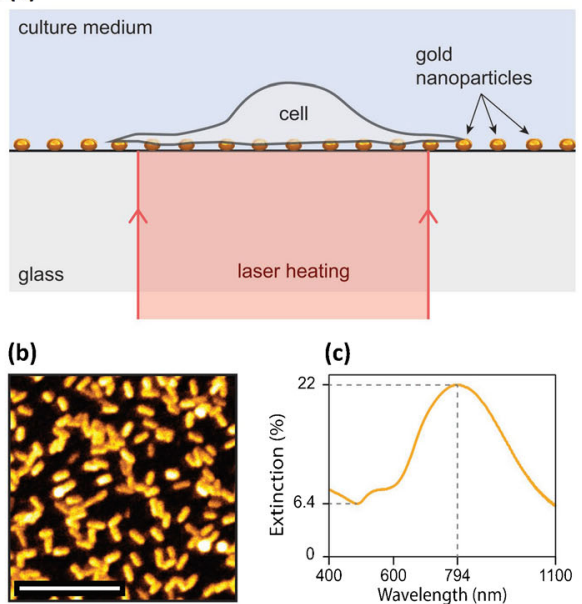
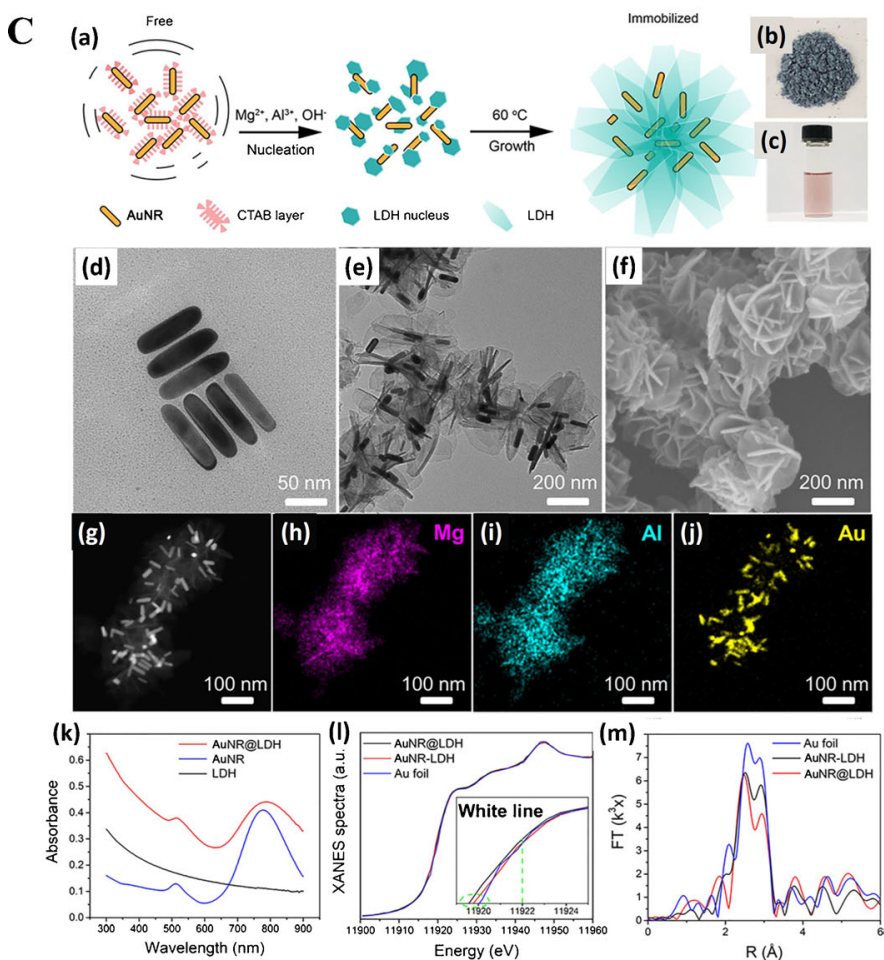

(n)
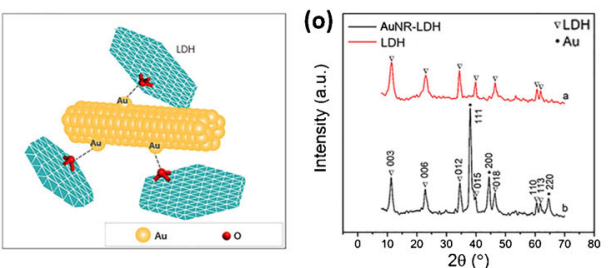

Fig. 5 A Schematic illustration of the AuNPs used as optical antennas for quantum biological electron tunneling imaging in a tunnel junction. Reprinted with permission from ref. [81], Copyright 2019 Springer Nature. B Description of the laser-induced dynamical control of the heat-shock response experimental approach. Reprinted with permission from ref. [82], Copyright 2018 WILEY-VCH Verlag GmbH \& Co. $\mathrm{KGaA}$, Weinheim. C Synthesis scheme and the performance parameter of AuNR@LDH. Reprinted with permission from ref. [83], Copyright 2019 American Chemical Society. D Schematic illustration of the self-
D

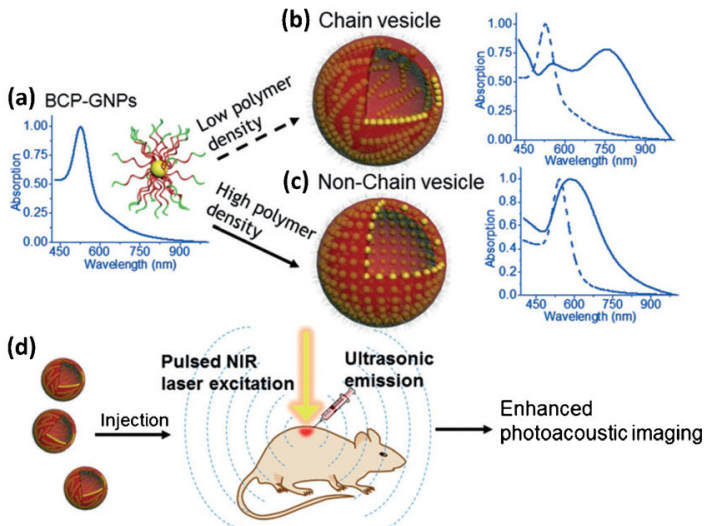

$\mathbf{E}$

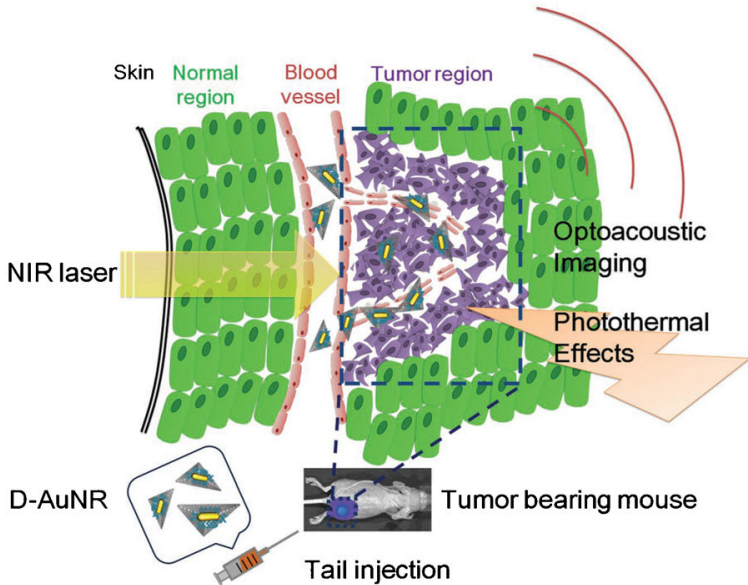

assembly of block copolymer-AuNPs into chain vesicles and nonchain vesicles, and the enhanced photoacoustic imaging with chain vesicles. Reprinted with permission from ref. [84], Copyright 2015 WILEYVCH Verlag GmbH \& Co. KGaA, Weinheim. E The graphical representation of the DNA-origami-AuNR hybrid (D-AuNR) nanoprobe system and the application of optoacoustic imaging and photothermal therapy. Reprinted with permission from ref. [85], Copyright 2016 WILEY-VCH Verlag GmbH \& Co. KGaA, Weinheim 
the AuNR@LDH could reach up to 60\% under 808 nm laser irradiation, which was a remarkable enhanced conversion efficiency as compared to those reported AuNR-based photothermal therapy materials $[94,95]$.

\section{Bioimaging based on photoacoustics}

Photoacoustics is another important technique for optical imaging in medical diagnosis because it takes advantage of sensitivity and selectivity of optical imaging, as well as deep penetration and high resolution of ultrasound imaging [96]. As described in the above section, photothermal imaging could provide nanoparticle tracking of high resolution; the photoacoustic imaging could offer both superb spatial resolution and deep penetration depth [97]. Studies have demonstrated that AuNPs can be used as contrast agent in photoacoustic imaging. For instance, Chen's group [84] developed an AuNP-assisted photoacoustic imaging method for biomedical applications (Fig. 5D). In their strategy, block copolymer (BCP) tethered AuNPs (BCPAuNPs) into hollow vesicles with membranes containing strings of AuNPs were stepwise self-assembled. The AuNP chain vesicles were modulated by adjusting the density of the polymer ligands on the surface of the AuNPs and showed obvious NIR absorption as contrast agents and drug delivery vehicles for photoacoustic imaging. Since these vesicles were stable under physiological conditions and conditions with slightly higher ionic strengths or lower $\mathrm{pH}$ values, great potential in bioimaging and drug delivery was revealed [98]. In the following year, the same group [99] reported the application of AuNPs as Raman probe, which could not only be applied to detect cancer cells, but also as the photoacoustic contrast agent for imaging-guided cancer therapy in two tumor xenograft models. In this assay, the AuNPs were coated on carbon nanotube ring (CNTR), and the CNTR acted as the template while surface-attached redox-active polymer as the reducing agent. Probably because of the combined effects of enhanced coupling between the embedded CNTR and the closely attached AuNPs plasmon mode, the extinction intensity ofCNTR@AuNPwas 120-fold higher than that of CNTR at $808 \mathrm{~nm}$, and the SERS signal of CNTR@ AuNP was $\sim 110$ times stronger than that of CNTR.

Other types of plasmonic gold nanostructures such as AuNRs, gold nanocages, and gold nanoshells have also been used in photoacoustic imaging. Taking AuNRs as an example, a nanoplatform has been constructed by $\mathrm{Du}$ et al. through assembling AuNRs on the surface of a DNA-origami structure (D-AuNR) [85]. As shown in Fig. 5E, by combining the AuNRs $(\sim 42 \mathrm{~nm} \times 12 \mathrm{~nm})$ and the DNA-origami structure, the resulted D-AuNR hybrid could serve as a distinct probe as well as a competent contrast agent in photoacoustic imaging and enable decreased dose and improved imaging quality. At the same time, the hybrid nanostructures responded to NIR irradiation for the photothermal therapy, effectively inhibited tumor regrowth, and prolonged the survival of diseased mice. Hajfathalian et al. [100] reported a multifunctional engineering of Wulff in cage gold-silver nanoparticles which could be used as photoacoustic imaging contrast agent, computed tomography, and potential agent for photothermal therapy. The Wulff-shaped nanostructure was formed by transferring the same shaped gold seeds into gold-silver core-shell structures to combine the advantages of both shell and core structures and perform a galvanic replacement reaction. To overcome the limitations of single mode diagnosis and treatment, Wang et al. [101] recently developed a magnetic resonance and photoacoustic dual-mode imaging-guided photothermalenhanced chemodynamic therapy through designing a gold@manganese dioxide $\left(\mathrm{Au} @ \mathrm{MnO}_{2}\right)$ core-shell nanostructure. Under the guidance of photoacoustic and magnetic resonance imaging, the $\mathrm{Au} @ \mathrm{MnO}_{2}$ nanostructure with a core size of $\sim 25 \mathrm{~nm}$ and a shell thickness of $\sim 14 \mathrm{~nm}$ could be activated by endogenous glutathione of a high concentration, exhibiting prominent synergistic treatment effect in photothermal-enhanced chemodynamic therapy.

\section{Conclusion and outlooks}

In this review, a number of selected examples have been highlighted to summarize the application of gold nanostructures in biosensing and bioimaging. For most of these selected examples, general mechanisms and major characteristics of them have been concluded and emphasized. It could be seen that due to the outstanding properties of the plasmonic gold nanostructures such as ease of functionalization and unique optical characteristics, numerous advantages including wide range of targets, high sensitivity, and rapid response have been shown by these examples. It is expected that these methods and strategies play a practical role in biosensing and bioimaging related area.

Since scientific research is about constant exploration, and there are still more effects are being put on this research topic, in our opinion, emphasis on the following aspects may prompt those forthcoming plasmonic gold nanostructures-based methods for biosensing and bioimaging closer to practical application.

(1) Besides sensitivity, specificity is another key performance of biosensing and bioimaging. Since the plasmonic gold nanostructures can be easily functionalized, probes of specific recognition ability such as antibodies, small peptides and aptamers could be conjugated with them to enable specific recognition of interested analytes $[102,103]$. Furthermore, it could be anticipated that through integrating appropriate specific recognition 
elements with the plasmonic gold nanostructures, multiplex detection of various interested analytes that is of great demands and broad prospects will be realized.

(2) Much efforts should be made to transfer the biosensing and bioimaging assays from lab bench to clinic [104]. In this respect, developing biosensing and bioimaging systems that possess good anti-interference ability as well as longer period of validity, it is highly required. Additionally, many more emerging materials as well as new signal conduction method should take into consideration.

(3) One of the ultimate goals in exploring novel biosensing and bioimaging strategies on basis of plasmonic gold nanostructures is applying them in early as well as precise diagnosis and treatment of diseases related to human health. Therefore, more concern should be put on minimizing the potential risks of those plasmonic gold nanostructures to human body. In this context, developing strategies such as the use of fewer gold nanostructures to achieve the same sensing and imaging effects, functionalizing gold nanostructures with natural proteins and PEG with low molecular for improving systematic clearance capabilities, and so on seems to be the reasonable development direction [105].

Funding This work is supported by National Natural Science Foundation of China (22004029, 32001785) and Scientific Research Project of Education Department of Hubei Province (Q20203003).

\section{Compliance with ethical standards}

Conflict of interest The authors declare that they have no competing of interests.

\section{References}

1. Stockman MI (2011) Nanoplasmonics: the physics behind the application. Phys Today 64:39-44

2. Amiens C, Ciuculescu-Pradines D, Philippot K (2016) Controlled metal nanostructures: fertile ground for coordination chemists. Coordin Chem Rev 308:409-432

3. Yuan-Fong CC, Chung-Ting CC, Hai-Pang C, Chee ML, Nyuk YV, Abdul HM (2018) Plasmonic effects in composite metal nanostructures for sensing applications. J Nanapart Res 20:190

4. Li S, Miao P, Zhang Y, Wu J, Zhang B, Du Y, Han X, Sun J, Xu P (2021) Recent advances in plasmonic nanostructures for enhanced photocatalysis and electrocatalysis. Adv Mat 33:2000086

5. Zhou W, Gao X, Liu D (2015) Gold nanoparticles for in vitro diagnostics. Chem Rev 19:10575-10636

6. Trantakis IA, Nilforoushan A, Dahlmann HA, Stäuble CK, Sturla $\mathrm{SJ}$ (2016) In-gene quantification of $\mathrm{O}_{6}$-methylguanine with elongated nucleoside analogues on gold nanoprobes. J Am Chem Soc 138:8497-8504

7. Wang K, Shangguan L, Liu Y, Jiang L, Zhang F, Wei Y, Zhang Y, Qi Z, Wang K, Liu S (2017) In situ detection and imaging of telomerase activity in cancer cell lines via disassembly of plasmonic core-satellites nanostructured probe. Anal Chem 89: $7262-7268$

8. Liu X, Zhang Y, Liang A, Ding H, Gai H (2019) Plasmonic resonance energy transfer from a Au nanosphere to quantum dots at a single particle level and its homogenous immunoassay. Chem Commun 55:11442-11445

9. Le NH, Ye G, Peng C, Chen J (2020) Metabolic mapping with plasmonic nanoparticle assemblies. Analyst 145:2586-2594

10. Le NH, Nguyen BK, Ye G, Peng C, Chen JIL (2017) Tuning the sensing performance of multilayer plasmonic core-satellite assemblies for rapid detection of targets from lysed cells. ACS Sensors 2:1578-1583

11. Ode K, Honjo M, Takashima Y, Tsuruoka T, Akamatsu K (2016) Highly sensitive plasmonic optical sensors based on gold coresatellite nanostructures immobilized on glass substrates. ACS Appl Mater Inter 8:20522-20526

12. Crut A, Maioli P, Del FN, Vallee F (2014) Optical absorption and scattering spectroscopies of single nano-objects. Chem Soc Rev 43:3921-3956

13. Deng X, Liang S, Cai X, Huang S, Cheng Z, Shi Y, Pang M, Ma PA, Lin J (2019) Yolk-Shell structured Au Nanostar@metal-organic framework for synergistic chemo-photothermal therapy in the second near-infrared window. Nano Lett 19:6772-6780

14. Fu Q, Zhu R, Song J, Yang H, Chen X (2018) Photoacoustic imaging: contrast agents and their biomedical applications. Adv Mater 6:1805875

15. Aldewachi H, Chalati T, Woodroofe MN, Bricklebank N, Sharrack B, Gardiner P (2018) Gold nanoparticle-based colorimetric biosensors. Nanoscale 10:18-33

16. Baetsen-Young AM, Vasher M, Matta LL, Colgan P, Alocilja EC, Day B (2018) Direct colorimetric detection of unamplified pathogen DNA by dextrin-capped gold nanoparticles. Biosens Bioelectron 101:29-36

17. Gao Z, Ye H, Tang D, Tao J, Habibi S, Minerick A, Tang D, Xia X (2017) Platinum-decorated gold nanoparticles with dual functionalities for ultrasensitive colorimetric in vitro diagnostics. Nano Lett 17:5572-5579

18. Koo Y, Lukianova-Hleb EY, Pan J, Thompson SM, Lapotko DO, Braam J (2016) In planta response of arabidopsis to photothermal impact mediated by gold nanoparticles. Small 12:623-630

19. Vines JB, Yoon J, Ryu N, Lim D, Park H (2019) Gold nanoparticles for photothermal cancer therapy. Front Chem 7:167

20. Chen C, Chang D, Li J, Chan H, Chen J, Chang C, Liu R, Chang CA, Chen C, Wang H (2020) Investigation of biodistribution and tissue penetration of PEGylated gold nanostars and their application for photothermal cancer treatment in tumor-bearing mice. $\mathrm{J}$ Mater Chem B 8:65-77

21. Sun L, Chen Y, Gong F, Dang Q, Xiang G, Cheng L, Liao F, Shao M (2019) Silicon nanowires decorated with gold nanoparticles via in situ reduction for photoacoustic imaging-guided photothermal cancer therapy. Journal of materials chemistry. B, Materials for Biology and Medicine 7:4393-4401

22. Wang J, Zhang Y, Jin N, Mao C, Yang M (2019) Protein-induced gold nanoparticle assembly for improving the photothermal effect in cancer therapy. ACS Appl Mater Inter 11:11136-11143

23. Huo D, Kim MJ, Lyu Z, Shi Y, Wiley BJ, Xia Y (2019) Onedimensional metal nanostructures: from colloidal syntheses to applications. Chem Rev 119:8972-9073

24. Mirkin CA, Letsinger RL, Mucic RC (1996) A DNA-based method for rationally assembling nanoparticles into macroscopic materials. Nature 382:607-609

25. Kim H, Park M, Hwang J, Kim JH, Chung D, Lee K, Kang M (2019) Development of label-free colorimetric assay for MERSCoV using gold nanoparticles. ACS Sensors 4:1306-1312

26. Liu B, Wu P, Huang Z, Ma L, Liu J (2018) Bromide as a robust backfiller on gold for precise control of DNA conformation and 
high stability of spherical nucleic acids. J Am Chem Soc 140: 4499-4502

27. Jeon J, Uthaman S, Lee J, Hwang H, Kim G, Yoo PJ, Hammock BD, Kim CS, Park Y, Park I (2018) In-direct localized surface plasmon resonance (LSPR)-based nanosensors for highly sensitive and rapid detection of cortisol. Sensors Actuators B Chem 266:710-716

28. Wei J, Chen H, Chen H, Cui Y, Qileng A, Qin W, Liu W, Liu Y (2019) Multifunctional peroxidase-encapsulated nanoliposomes: bioetching-induced photoelectrometric and colorimetric immunoassay for broad-spectrum detection of ochratoxins. ACS Appl Mater Inter 11:23832-23839

29. Ren W, Ballou DR, FitzGerald R, Irudayaraj J (2019) Plasmonic enhancement in lateral flow sensors for improved sensing of $\mathrm{E}$. coli O157:H7. Biosens Bioelectron 126:324-331

30. Wang C, Zhang X, Liu K, Dai X, Yang C, Guo S, Su H (2019) Triplet excited state enhancement induced by PDDA polymerassembled gold nanoparticles. J Phys Chem C 123:27717-27724

31. Pacioni NL, Gonzalez-Bejar M, Alarcon E, McGilvray KL, Scaiano JC (2010) Surface plasmons control the dynamics of excited triplet states in the presence of gold nanoparticles. J Am Chem Soc 132:6298-6299

32. Yang W, Liu K, Song D, Du Q, Wang R, Su H (2013) Aggregation-induced enhancement effect of gold nanoparticles on triplet excited state. J Phys Chem C 117(51):27088-27095

33. Rao A, Kumar GS, Roy S, Rajesh AT, Devatha G, Pillai PP (2019) Turn-on selectivity in inherently nonselective gold nanoparticles for $\mathrm{Pb}^{2+}$ detection by preferential breaking of interparticle interactions. ACS Applied Nano Materials 2:5625-5633

34. Funari R, Chu K, Shen AQ (2020) Detection of antibodies against SARS-CoV-2 spike protein by gold nanospikes in an optomicrofluidic chip. Biosens Bioelectron 169:112578

35. Lin T, Zhang M, Xu F, Wang X, Xu Z, Guo L (2018) Colorimetric detection of benzoyl peroxide based on the etching of silver nanoshells of au@ag nanorods. Sensors Actuators B Chem 261:379384

36. Mishra RK, Hayat A, Catanante G, Istamboulie G, Marty JL (2016) Sensitive quantitation of Ochratoxin A in cocoa beans using differential pulse voltammetry based aptasensor. Food Chem 192:799-804

37. Zejli H, Goud KY, Marty JL (2018) Label free aptasensor for ochratoxin a detection using polythiophene-3-carboxylic acid. Talanta 185:513-519

38. Storhoff JJ, Lucas AD, Garimella V, Bao YP, Müller UR (2004) Homogeneous detection of unamplified genomic DNA sequences based on colorimetric scatter of gold nanoparticle probes. Nat Biotechnol 22:883-887

39. Zhai T, Ye D, Shi Y, Zhang Q, Qin X, Wang C, Xia X (2018) Plasmon coupling effect-enhanced imaging of metal ions in living cells using DNAzyme assembled core-satellite structures. ACS Appl Mater Inter 10:33966-33975

40. Zhao Y, Gao X, Wang H, Wang J, Zhou J, Zhao W, Xu J, Chen H (2019) Ultrasensitive detection of MicroRNA via a Au@Ag nanosnowman. Anal Chem 91:15988-15992

41. Li S, Kong Q, Zhang M, Yang F, Kang B, Xu J, Chen H (2018) Plasmon-resonance-energy-transfer-based spectroscopy on single nanoparticles: biomolecular recognition and enzyme kinetics. Anal Chem 90:3833-3841

42. Li J, Wu J, Zhang X, Liu Y, Zhou D, Sun HZ, Zhang H, Yang B (2011) Controllable synthesis of stable urchin-like gold nanoparticles using hydroquinone to tune the reactivity of gold chloride. J Phys Chem C 115:3630-3637

43. Shi Y, Wang R, Yuan W, Liu Q, Shi M, Feng W, Wu Z, Hu K, Li F (2018) Easy-to-use colorimetric cyanine probe for the detection of $\mathrm{Cu}^{2+}$ in Wilson's disease. ACS Appl Mater Inter 10(24):2037720386
44. Sarkar S, Chatti M, Adusumalli VNKB, Mahalingam V (2015) Highly selective and sensitive detection of $\mathrm{Cu}^{2+}$ ions using $\mathrm{Ce}$ (III)/ $\mathrm{Tb}(\mathrm{III})$-doped $\mathrm{SrF} 2$ nanocrystals as fluorescent probe. Appl Mater Inter 7(46):25702-25708

45. Waldeisen JR, Wang T, Ross BM, Lee LP (2011) Disassembly of a core-satellite nanoassembled substrate for colorimetric biomolecular detection. ACS Nano 5:5383-5389

46. Yao B, Liu Y, Tabata M, Zhua H, Miyahara Y (2014) Sensitive detection of microRNA by chronocoulometry and rolling circle amplification on a gold electrode. Chem Commun 50:9704-9706

47. Hwu S, Blickenstorfer Y, Tiefenauer RF, Gonnelli C, Schmidheini L, Lüchtefeld I, Hoogenberg B, Gisiger AB, Vörös J (2019) Dark-field microwells toward high-throughput direct miRNA sensing with gold nanoparticles. ACS Sensors 4:19501956

48. Gao PF, Li YF, Huang CZ (2020) Plasmonics-attended NSET and PRET for analytical applications. TrAC Trends Anal Chem 124: 115805

49. Liu XG, Long YT, Choi Y, Wang T, Lee LP (2007) Quantized plasmon quenching dips nanospectroscopy via plasmon resonance energy transfer. Nat Methods 4:1015-1017

50. Xin HB, Namgung B, Lee LP (2018) Nanoplasmonic optical antennas for life sciences and medicine. Nat Rev Mater 3:228-243

51. Yan X, Xia C, Chen B, Li YF, Gao PF, Huang CZ (2020) Enzyme activity triggered blocking of plasmon resonance energy transfer for highly selective detection of acid phosphatase. Anal Chem 92: 2130-2135

52. Wang J, Li X, Chen H, Xu J (2020) "Loading-type" plasmonic nanoparticles for detection of peroxynitrite in living cells. Anal Chem 92:15647-15654

53. Ma J, Gao MX, Zuo H, Li YF, Gao PF, Huang CZ (2020) Distance-dependence study of plasmon resonance energy transfer with DNA spacers. Anal Chem 92:14278-14283

54. Lakowicz JR (2005) Radiative decay engineering 5: metalenhanced fluorescence and plasmon emission. Anal Biochem 337:171-194

55. Gao Y, Wang J, Wang W, Zhao T, Cui Y, Liu P, Xu S, Luo X (2021) More symmetrical "hot spots" ensure stronger plasmonenhanced fluorescence: from $\mathrm{Au}$ nanorods to nanostars. Anal Chem 93(4:2480-2489

56. Peng M, Sun F, Na N, Ouyang J (2020) Target-triggered assembly of nanogap antennas to enhance the fluorescence of single molecules and their application in MicroRNA detection. Small 16: 2000460

57. Luo X, Zhao X, Wallace GQ, Brunet MH, Wilkinson KJ, Wu P, Cai C, Bazuin G, Masson JF (2021) Multiplexed SERS detection of microcystins with aptamer-driven core-satellite assemblies. ACS Appl Mater Interfaces 13:6545-6556

58. Zhang H, Lai H, Wu X, Li G, Hu Y (2020) $\mathrm{CoFe}_{2} \mathrm{O}_{4} @ \mathrm{HNTs} /$ AuNPs substrate for rapid magnetic solid-phase extraction and efficient SERS detection of complex samples all-in-one. Anal Chem 92:4607-4613

59. Wang A, Lin Q, Liu S, Li J, Wang J, Quan K, Yang X, Huang J, Wang K (2020) Aptamer-tethered self-assembled FRET-flares for microRNA imaging in living cancer cells. Chem Commun 56(16): 2463-2466

60. Xiao H, Fan H, Xu L, Pei Z, Lei SB, Xu J, Xi J, Wang G, Wang L, Wang Z (2019) Gold-nanodot-decorated hollow carbon nanosphere based nanoplatform for intracellular miRNA imaging in colorectal cancer cells. Chem Commun 55:12352-12355

61. Zhou L, Chen K, Ramsey JD, Almlie CK, Burrows SM (2018) Lighting up plasmonic nanostar colloids for metal-enhanced fluorescence under two-photon near-infrared excitation. J Phys Chem C 122:19823-19830

62. Miranda B, Chu K, Maffettone PL, Shen AQ, Funari R (2020) Metal-enhanced fluorescence immunosensor based on plasmonic 
arrays of gold nanoislands on an etched glass substrate. ACS Applied Nano Materials 3:10470-10478

63. Vlug A, Nieuwenhuys EJ, Van Eijk RVW, Geertzen HGM, Van Houte AJ (1994) Nephelometric measurements of human IgG subclasses and their reference ranges. Ann Biol Clin 52(7-8): $561-567$

64. Xia Y, Wang L, Li J, Chen X, Lan J, Yan A, Lei Y, Yang S, Yang H, Chen J (2018) A ratiometric fluorescent bioprobe based on carbon dots and acridone derivate for signal amplification detection exosomal microRNA. Anal Chem 90(15):8969-8976

65. Theodorou IG, Ruenraroengsak P, Gonzalez-Carter DA, Jiang Q, Yague E, Aboagye EO, Coombes RC, Porter AE, Ryan MP, Xie F (2019) Towards multiplexed near-infrared cellular imaging using gold nanostar arrays with tunable fluorescence enhancement. Nanoscale 11:2079-2088

66. Tabakman SM, Chen Z, Casalongue HS, Wang H, Dai H (2011) A new approach to solution-phase gold seeding for SERS substrates. Small 7:499-505

67. Luan J, Seth A, Gupta R, Wang Z, Rathi P, Cao S, Gholami Derami H, Tang R, Xu B, Achilefu S, Morrissey JJ, Singamaneni S (2020) Ultrabright fluorescent nanoscale labels for the femtomolar detection of analytes with standard bioassays. Nat Biomed Eng. https://doi.org/10.1038/s41551-020-0547-4

68. Chakkarapani SK, Lee S, Park B, Seo H, Kang SH (2019) Plasmon-amplified endogenous fluorescence nanospectroscopic sensor based on inherent elastic scattering for ultratrace ratiometric detection of capsaicinoids. ACS Sensors 4:953-960

69. Zak A, Siwinska N, Slowikowska M, Borowicz H, Szpot P, Zawadzki M, Niedzwiedz A (2018) The detection of capsaicin and dihydrocapsaicin in horse serum following long-term local administration. BMC Vet Res 14(1):1-6

70. Zong C, Xu M, Xu L, Wei T, Ma X, Zheng X, Hu R, Ren B (2018) Surface-enhanced raman spectroscopy for bioanalysis: reliability and challenges. Chem Rev 118:4946-4980

71. Zong S, Chen C, Wang Z, Zhang Y, Cui Y (2016) Surface enhanced raman scattering based in situ hybridization strategy for telomere length assessment. ACS Nano 10:2950-2959

72. Wei Q, Zhao Y, Du B, Wu D, Cai Y, Mao K, Li H, Xu C (2011) Nanoporous $\mathrm{PtRu}$ alloy enhanced nonenzymatic immunosensor for ultrasensitive detection of microcystin-LR. Adv Funct Mater 21:4193-4198

73. Feng E, Zheng T, Tian Y (2018) Dual-mode Au nanoprobe based on surface enhancement Raman scattering and colorimetry for sensitive determination of telomerase activity both in cell extracts and in the urine of patients. ACS Sensors 4:211-217

74. Choi N, Dang H, Das A, Sim MS, Chung IY, Choo J (2020) SERS biosensors for ultrasensitive detection of multiple biomarkers expressed in cancer cells. Biosens Bioelectron 164:112326

75. Hong S, Shim O, Kwon H, Choi Y (2016) Autoenhanced raman spectroscopy via plasmonic trapping for molecular sensing. Anal Chem 88:7633-7638

76. Kuttner C, Mayer M, Dulle M, Moscoso A, Lopez-Romero JM, Forster S, Fery A, Perez-Juste J, Contreras-Caceres R (2018) Seeded growth synthesis of gold nanotriangles: size control, SAXS analysis, and SERS performance. ACS Appl Mater Interfaces 10:11152-11163

77. Scarabelli L, Coronado-Puchau M, Giner-Casares JJ, Langer J, Liz-Marzán LM (2014) Monodisperse gold nanotriangles: size control, large-scale self-assembly, and performance in surfaceenhanced raman scattering. ACS Nano 8(6):5833-5842

78. Park JE, Kim M, Hwang JH (2017) Golden opportunities: plasmonic gold nanostructures for biomedical applications based on the second near-infrared window. Small 1:1600032

79. Lee K, Cui Y, Lee LP, Irudayaraj J (2014) Quantitative imaging of single mRNA splice variants in living cells. Nat Nanotechnol 9: 474-480
80. Park S, Aalipour A, Vermesh O, Yu JH, Gambhir SS (2017) Towards clinically translatable in vivo nanodiagnostics. Nat Rev Mater 2(5): 1-20

81. Xin H, Sim WJ, Namgung B, Choi Y, Li B, Lee LP (2019) Quantum biological tunnel junction for electron transfer imaging in live cells. Nat Commun 10:3245-3256

82. Robert HML, Savatier J, Vial S, Verghese J, Wattellier B, Rigneault H, Monneret S, Polleux J, Baffou G (2018) Photothermal control of heat-shock protein expression at the single cell level. Small 14:1801910

83. Ma K, Li Y, Wang Z, Chen Y, Zhang X, Chen C, Yu H, Huang J, Yang Z, Wang X, Wang Z (2019) Core-shell gold nanorod@layered double hydroxide nanomaterial with highly efficient photothermal conversion and its application in antibacterial and tumor therapy. ACS Appl Mater Inter 11:29630-29640

84. Liu Y, He J, Yang K, Yi C, Liu Y, Nie L, Khashab NM, Chen X, Nie Z (2015) Folding up of gold nanoparticle strings into plasmonic vesicles for enhanced photoacoustic imaging. Angew Chem Inter Ed 54:15809-15812

85. Du Y, Jiang Q, Beziere N, Song L, Zhang Q, Peng D, Chi C, Yang X, Guo H, Diot G, Ntziachristos V, Ding B, Tian J (2016) DNAnanostructure-gold-nanorod hybrids for enhanced in vivo optoacoustic imaging and photothermal therapy. Adv Mater 28: 10000-10007

86. Goh BH, Tong ES, Pusparajah P (2020) Quantum biology: does quantum physics hold the key to revolutionizing medicine? Prog Drug Discov Biomed Sci 3(1)

87. Cho H, Hossain MK, Lee J, Han J, Lee HJ, Kim K, Kim J, Lee K, Choi J (2018) Selective isolation and noninvasive analysis of circulating cancer stem cells through Raman imaging. Biosens Bioelectron 102:372-382

88. Younis MR, Wang C, An R, Wang S, Younis MA, Li Z, Wang Y, Ihsan A, Ye D, Xia X (2019) Low power single laser activated synergistic cancer phototherapy using photosensitizer functionalized dual plasmonic photothermal nanoagents. ACS Nano 13: 2544-2557

89. Lasne D, Blab GA, Berciaud S, Heine M, Groc L, Choquet D, Cognet L, Lounis B (2006) Single nanoparticle photothermal tracking $(\mathrm{SNaPT})$ of 5-nm gold beads in live cells. Biophys J 91: 4598-4604

90. Jauffred L, Samadi A, Klingberg H, Bendix PM, Oddershede LB (2019) Plasmonic heating of nanostructures. Chem Rev 119(13): 8087-8130

91. Shibu ES, Varkentina N, Cognet L, Lounis B (2017) Small gold nanorods with tunable absorption for photothermal microscopy in cells. Adv Sci 4:1600280

92. Kang H, Buchman JT, Rodriguez RS (2019) Stabilization of silver and gold nanoparticles: preservation and improvement of plasmonic functionalities. Chem Rev 1:664-699

93. Liu T, Shen S, Huang Y, Zhang X, Lai Z, Tran TH, Liu Z, Cheng $\mathrm{L}$ (2019) Controllable growth of $\mathrm{Au}$ nanostructures onto MoS2 nanosheets for dual-modal imaging and photothermal-radiation combined therapy. Nanoscale 11:22788-22795

94. Wang B, Wang JH, Liu Q, Huang H, Chen M, Li K, Li C, Yu XF, Chu PK (2014) Rose-bengal-conjugated gold nanorods for in vivo photodynamic and photothermal oral cancer therapies. Biomaterials 35:1954-1966

95. Li G, Chen Y, Zhang L, Zhang M, Li S, Li L, Wang T, Wang C (2018) Facile approach to synthesize gold nanorod@polyacrylic acid/calcium phosphate yolk-shell nanoparticles for dual-mode imaging and $\mathrm{pH} / \mathrm{NIR}$-responsive drug delivery. Nano-Micro Lett 10(1):7

96. Cui D, Li P, Zhen X (2019) Thermoresponsive semiconducting polymer nanoparticles for contrast-enhanced photoacoustic imaging. Adv Funct Mater 29:1903461 
97. Fathi P, Knox HJ, Sar D (2019) Biodegradable biliverdin nanoparticles for efficient photoacoustic imaging. ACS Nano 13:76907704

98. Yi C, Yang Y, Liu B, He J, Nie Z (2020) Polymer-guided assembly of inorganic nanoparticles. Chem Soc Rev 49(2):465-508

99. Song J, Wang F, Yang X, Ning B, Harp MG, Culp SH, Hu S, Huang P, Nie L, Chen J, Chen X (2016) Gold nanoparticle coated carbon nanotube ring with enhanced Raman scattering and photothermal conversion property for theranostic applications. J Am Chem Soc 138:7005-7015

100. Hajfathalian M, Amirshaghaghi A, Naha PC, Chhour P, Hsu JC, Douglas K, Dong Y, Sehgal CM, Tsourkas A, Neretina S, Cormode DP (2018) Wulff in a cage gold nanoparticles as contrast agents for computed tomography and photoacoustic imaging. Nanoscale 10:18749-18757

101. Wang H, An L, Tao C, Ling Z, Lin J, Tian Q, Yang S (2020) A smart theranostic platform for photoacoustic and magnetic resonance dual-imaging-guided photothermal-enhanced chemodynamic therapy. Nanoscale 12:5139-5150

102. Zhou F, Fu T, Huang Q (2019) Hypoxia-activated PEGylated conditional aptamer/antibody for cancer imaging with improved specificity. J Am Chem Soc 141:18421-18427

103. Peng L, Li BL, Zhou CW, Li NB, Setyawati MI, Zou HL (2018) "Naked-eye" recognition: emerging gold nano-family for visual sensing. Appl Mater Today 11:166-188

104. Rudra A, Li J, Shakur R (2020) Trends in therapeutic conjugates: bench to clinic. Bioconjug Chem 31:462-473

105. Fu Q, Zhang X, Song J, Yang HH (2021) Plasmonic gold nanoagents for cancer imaging and therapy. View 20200149

Publisher's note Springer Nature remains neutral with regard to jurisdictional claims in published maps and institutional affiliations. 